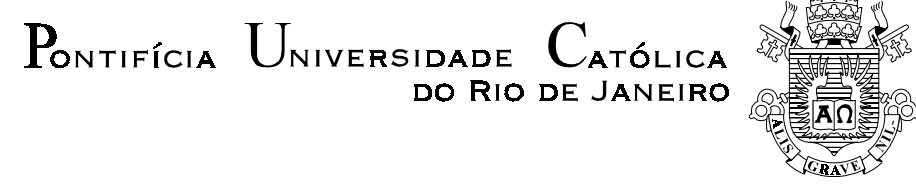

Maurício Cortez Reis

Mudanças no desemprego por nível de qualificação durante a década de noventa no Brasil

Tese apresentada como requisito parcial para obtenção do título de Doutor pelo Programa de Pós-Graduação em Economia da PUC-Rio.

Orientador: Gustavo Gonzaga Co-orientador: José Márcio Camargo

Rio de janeiro, março de 2004 


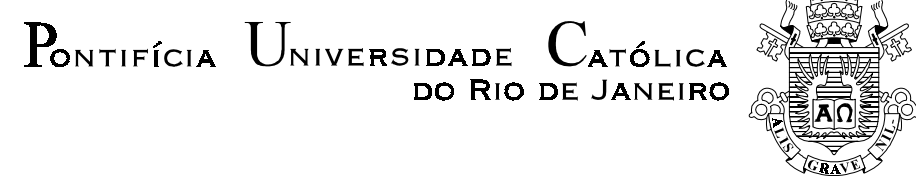

Maurício Cortez Reis

\title{
Mudanças no desemprego por nível de qualificação durante a década de noventa no Brasil
}

\begin{abstract}
Tese apresentada como requisito parcial para obtenção do título de Doutor pelo Programa de Pós-Graduação em Economia da PUC-Rio. Aprovada pela Comissão Examinadora abaixo assinada.
\end{abstract}

\author{
Gustavo Gonzaga \\ Orientador \\ PUC-Rio \\ José Márcio Camargo \\ Co-orientador \\ PUC-Rio \\ Ricardo Paes de Barros \\ IPEA \\ Eduardo Rios-Neto \\ UFMG \\ Reynaldo Fernandes \\ ESAF e USP-RP \\ João Pontes Nogueira
}

Coordenador(a) Setorial do Centro de Ciências Sociais - PUC-Rio

Rio de Janeiro, 24 de março de 2004 
Todos os direitos reservados. É proibida a reprodução total ou parcial do trabalho sem autorização da universidade, do autor e do orientador.

\section{Maurício Cortez Reis}

Graduou-se em economia pela Universidade do Estado do

Rio de Janeiro (UERJ). Especializou-se nas áreas de Economia do Trabalho e Economia do setor Público pela

Pontifícia Universidade Católica do Rio de Janeiro (PUC-

Rio). Atualmente é técnico de pesquisa do Instituto de

Pesquisa Econômica Aplicada (IPEA)

Ficha Catalográfica

Reis, Maurício Cortez

Mudanças no desemprego por nível de qualificação durante a década de noventa no Brasil

v.; 195 f: il. ; $29 \mathrm{~cm}$

Tese de doutorado

Incluí referências bibliográficas.

Desemprego; nível de qualificação; mudanças tecnológicas; decomposição por idade, período e coorte; aposentadoria

CDD:330 


\section{Agradecimentos}

Ao meu orientador, Prof. Gustavo Gonzaga, pela dedicação e paciência, que foram fundamentais durante todo o processo de elaboração da tese.

Ao meu co-orientador, Prof. José Márcio Camargo, pelas inúmeras discussões, sempre muito enriquecedoras, sobre o mercado do trabalho.

Aos meus professores durante o curso de doutorado: Afonso Bevilaqua, Eduardo Loyo, Fábio Kanczuk, Francisco Ferreira, José Márcio Camargo, Marcelo Abreu, Márcio Garcia, Naércio Menezes-Filho, Rogério Werneck e Thierry Magnac.

Aos membros da comissão examinadora, Ricardo Paes de Barros, Eduardo RiosNeto e Reynaldo Fernandes, pelas inúmeras sugestões.

A todos os amigos com que convivi durante o curso de doutorado. Em especial para Anna, Antonio, Dani, Daniel Santos, Emílio, Fernanda e Kiko.

Aos meus pais e ao meu irmão pelo apoio.

A Graça, pela ajuda nas questões administrativas.

A CAPES e ao CNPQ pelo apoio financeiro. 


\section{Resumo}

Reis, Maurício Cortez. Mudanças no desemprego por nível de qualificação durante a década de noventa no Brasil. Rio de janeiro, 2004. 195p. Tese de Doutorado - Departamento de Economia, Pontifícia Universidade Católica do Rio de Janeiro.

A taxa de desemprego no Brasil aumentou significativamente durante os anos noventa. Nota-se também que esse aumento do desemprego foi muito mais intenso para os trabalhadores com níveis mais baixos de qualificação. O objetivo desta tese é identificar os fatores que determinaram essas diferentes trajetórias da taxa de desemprego entre os grupos de qualificação. A tese é composta de seis capítulos, incluindo a introdução e a conclusão. Os resultados encontrados nos capítulos 2 e 3 mostram que ocorreu um importante aumento na produtividade dos trabalhadores qualificados em relação aos semi-qualificados e os nãoqualificados durante a década de noventa. Esse resultado parece estar associado à utilização de tecnologias mais modernas, intensivas em trabalho qualificado, após a liberalização comercial. Com isso, a taxa de desemprego dos trabalhadores qualificados teria diminuído em relação aos demais grupos. O capítulo 4 mostra que apesar do desemprego dos trabalhadores qualificados ter diminuído em relação aos demais grupos de qualificação nas coortes mais novas, nas gerações mais antigas o desemprego relativo dos qualificados aumentou. Decompondo o comportamento do desemprego por nível de qualificação em efeitos associados à idade, ao período e a coorte, são encontradas evidências de que características específicas das gerações mais novas de trabalhadores qualificados contribuíram para a redução no desemprego relativo desse grupo. Esse resultado é consistente com a hipótese de que o progresso tecnológico deve ter favorecido os trabalhadores qualificados das gerações mais novas, em função das características da educação recebida por esse grupo. O capítulo 5 apresenta evidências de que aumentos na pressão salarial também foram importantes para o comportamento do desemprego por nível de qualificação. Durante os anos noventa, ocorreram aumentos na aposentadoria domiciliar per capita, 
principalmente para os trabalhadores não-qualificados. De acordo com o argumento proposto no capítulo, a maior renda decorrente da aposentadoria teria aumentado o salário de reserva dos trabalhadores, gerando um aumento da pressão salarial, o que, por sua vez, teria levado a maiores taxas de desemprego. Os resultados empíricos mostram que maiores aposentadorias estão diretamente relacionadas a taxas mais altas de desemprego para os trabalhadores nãoqualificados.

\section{Palavras-chave}

Desemprego; nível de qualificação; mudanças tecnológicas; decomposição por idade, período e coorte; aposentadoria. 


\section{Abstract}

Reis, Maurício Cortez. Changes in Unemployment by skill: evidence from Brazil during the nineties. Rio de janeiro, 2004. 195p. PhD. Thesis - Departamento de Economia, Pontifícia Universidade Católica do Rio de Janeiro.

During the nineties, Brazil experienced a remarkable increase in its unemployment rate. The rise in unemployment was more intense for unskilled and semi-skilled workers relative to skilled workers. The objective of this thesis is to identify the determinants for the different patterns of unemployment rate by skill during the nineties. The thesis is organized into six chapters, including the introduction and the conclusion. The chapters 2 and 3 of the thesis show that occurred an increase of skilled workers productivity relative to unskilled and semi-skilled during the nineties, which is consistent with the technological progress that followed the trade liberalization. As a consequence of these changes, the skilled workers unemployment rate decreased relative to the other groups. The chapter 4 presents evidences that the skilled relative unemployment rate decreased for the younger skilled cohorts, while older skilled cohorts experienced increases in its relative unemployment rate. The empirical evidence, obtained decomposing the unemployment rate by skill into age, period and cohort effects, shows that characteristics associated with younger skilled cohorts contributed for the reduction in this group unemployment rate. This could be explained by the fact that technological changes in the nineties were biased toward these workers because of their type and quality of education. The chapter 5 shows that increases in the wage pressure were important to the relative unemployment rate pattern. During the nineties the household retirement income per capita rose sharply in Brazil, and it happened in a more intense way for the unskilled workers than for the other groups. According to the argument of this chapter, the household retirement income increasing affected workers reservation wage, which augmented the wage pressure. Consequently, the higher wage pressure implied in larger unemployment rate. The empirical evidence 
shows that the household retirement income is positively associated with the unemployment rate for unskilled workers.

\section{Keywords}

Unemployment; Skills; technological change, decomposition by age, period and cohort; retirement. 


\section{Sumário}

1 Introdução

2 Os impactos das mudanças na demanda e na oferta de trabalho qualificado sobre o desemprego por nível de qualificação. 20

2.1. Introdução 20

2.2. Revisão da literatura 22

2.3. Fatos estilizados 38

2.4. O modelo 45

2.5. Resultados empíricos $\quad 53$

2.6. Os impactos de choques agregados e relativos sobre o desemprego 59

$\begin{array}{ll}\text { 2.7. Conclusão } & 65\end{array}$

3 Decompondo as mudanças no desemprego e nos rendimentos $\begin{array}{ll}\text { por nível de qualificação } & 67\end{array}$

3.1. Introdução 67

3.2. Análise descritiva dos dados 68

$\begin{array}{ll}\text { 3.3. Abordagem teórica } & 74\end{array}$

3.4. Resultados 82

$\begin{array}{ll}\text { 3.5. Conclusão } & 87\end{array}$

4 Desemprego e qualificação: uma análise dos efeitos idade, período e coorte. 89

4.1. Introdução 89

4.2. Abordagem teórica 92

4.3. Os dados 96

4.4. A evolução do desemprego relativo por idade, período e coorte 98

4.5. Estratégia empírica 104

$\begin{array}{ll}\text { 4.6. Resultados } & 106\end{array}$ 
5 Aposentadoria, pressão salarial e desemprego 120

5.1. Introdução 120

5.2. Efeitos da aposentadoria sobre o desemprego e a participação: teoria1

5.3. Dados

5.4. Análise descritiva 132

5.5. Metodologia 142

5.6. Evidências empíricas para os efeitos da aposentadoria domiciliar per capita sobre o desemprego

5.7. Evidências empíricas para as taxas de participação e de nãoemprego

5.8. Conclusão

6 Conclusão

7 Referências bibliográficas

8 Apêndice

8.1. Apêndice do capítulo 2

8.2. Apêndice do capítulo 3

8.3. Apêndice do capítulo 4

181

8.4. Apêndice do capítulo 5 


\section{Lista de figuras}

Figura 1-Taxa de desemprego por nível de escolaridade (\%) 39

Figura 2-Evolução do total de empregados por nível de escolaridade 43

Figura 3-Diferenças entre as taxas de desemprego por qualificação 69

$\begin{array}{ll}\text { Figura 4-Rendimentos relativos por qualificação } & 70\end{array}$

Figura 5-Mudanças no emprego e no nível de qualificação entre os setores: 1990-1999 73

Figura 6-Taxa de desemprego por coorte de nascimento (\%) 100

Figura 7-Taxa de desemprego por coorte para cada grupo de qualificação (\%) 100

Figura 8-Diferenças entre as taxas de desemprego nas coortes

(qualificados x semi-qualificados) 102

Figura 9-Diferenças entre as taxas de desemprego nas coortes

(qualificados x não-qualificados) 103

Figura 10-Participação na PEA por coorte e qualificação: 1999

Figura 11-Efeitos idade, período e coorte: qualificados x semi-qualificados 108

Figura 12-Efeitos idade, período e coorte: qualificados x não-qualificados 111

Figura 13- Efeitos idade, período e coorte para as regressões com polinômios 185

Figura 14- Efeitos idade, período e coorte para o modelo com

polinômios incluindo interações entre coorte e idade

Figura 15- Efeitos idade, período e coorte para o modelo com polinômios incluindo interações entre coorte e período

Figura 16- Efeitos idade, período e coorte para as diferenças das probabilidade de desemprego 


\section{Lista de tabelas}

Tabela 1-Taxas de desemprego por educação dos homens em oito países da OCDE. 25

Tabela 2-Taxa de desemprego por nível de escolaridade (\%) 40

Tabela 3-Rendimento médio do trabalho principal por nível de escolaridade (R\$ de 1999) 41

Tabela 4-Composição da população economicamente ativa por nível de escolaridade (\%) 42

Tabela 5-Participação dos grupos educacionais no total de gastos com os rendimentos do trabalho principal (\%) 43

Tabela 6-Taxa de desemprego prevista sem mudanças na PEA (\%) 45

Tabela 7 - Curvas de salário por nível de qualificação 55

Tabela 8-Elasticidade-substituição entre qualificados e não-qualificados 58

Tabela 9-Efeitos de choques agregados sobre a variação do desemprego por qualificação: 1990-1999 60

Tabela 10-Variações dos parâmetros de produtividade por qualificação: 19901999

Tabela 11-Efeitos de variações nos parâmetros de produtividade e na composição da PEA sobre o desemprego: 1990 - 1999

Tabela 12-Efeitos totais de choques na produtividade relativa e na composição da oferta sobre as variações no desemprego por qualificação: 1990-1999 63

Tabela 13 - Contribuições de choques relativos para o aumento do desemprego agregado e por qualificação: 1990-1999

Tabela 14-Desemprego, rendimentos, participações na PEA e nos gastos com rendimentos por qualificação $\quad 69$

Tabela 15-Emprego e qualificação por setor em 1990 e 1999

Tabela 16-Decomposição da variação no emprego por nível de qualificação: 1990-1999

Tabela 17-Decomposições das variações do desemprego relativo: 1990-1999 83

Tabela 18-Decomposições das variações dos rendimentos relativos: 1990-199985

Tabela 19-Classificação das coortes pela idade e o ano da pesquisa 
Tabela 20-Taxa de desemprego por qualificação (\%)

Tabela 21-Participação na força de trabalho por qualificação (\%) 99

Tabela 22-Taxa de desemprego por qualificação e grupo etário (\%) 101

Tabela 23-Efeitos idade, período e coorte sobre o desemprego relativo 107

Tabela 24-Efeitos idade, período e coorte sobre o desemprego relativo 109

Tabela 25-Taxas de desemprego (\%): 1981-1999 133

Tabela 26-Taxas de desemprego por qualificação (\%): 1981-1999 134

Tabela 27-Aposentadoria domiciliar per capita para os domicílios com indivíduos entre 25 e 59 anos na PEA (em R \$ de 1999) 136

Tabela 28-Aposentadoria - valor médio e porcentagem dos indivíduos que recebem renda de aposentadoria

Tabela 29-Porcentagem que recebe aposentadoria em grupos de idade e participação na PEA 138

Tabela 30-Rendimento médio do trabalho principal e participação da aposentadoria na renda domiciliar 139

Tabela 31-Taxa de participação por qualificação (\%): 1981-1999 141

Tabela 32-Efeitos da aposentadoria sobre a taxa de desemprego (não-qualificados)

148

Tabela 33- Efeitos da aposentadoria sobre a taxa de desemprego (semiqualificados)

148

Tabela 34- Efeitos da aposentadoria sobre a taxa de desemprego (qualificados) 149

Tabela 35-Efeitos da aposentadoria sobre a taxa de desemprego de longo prazo

Tabela 36- Efeitos da aposentadoria sobre a proporção de desempregados de longo prazo

Tabela 37-Efeitos da aposentadoria sobre a taxa de desemprego: modelo autoregressivo

Tabela 38- Efeitos da aposentadoria sobre a taxa de desemprego de longo prazo: modelo auto-regressivo

Tabela 39-Efeitos da aposentadoria sobre o desemprego com a amostra completa

Tabela 40-Regressões logit para a probabilidade de desemprego

Tabela 41- Regressões logit para a probabilidade de desemprego de longo prazo 
Tabela 42-Efeitos da aposentadoria sobre a taxa de participação 158

Tabela 43- Efeitos da aposentadoria sobre a taxa de não-emprego 158

Tabela 44-Regressões logit para a probabilidade de participar da PEA 159

Tabela 45- Regressões logit para a probabilidade de não-emprego 160

Tabela 46-Taxas de desemprego na OCDE (\%) 173

Tabela 47-Efeitos de choques agregados sobre a variação do desemprego por qualificação: 1990-1999 173

Tabela 48- Variações dos parâmetros de produtividade por qualificação: 19901999

Tabela 49-Efeitos de variações nos parâmetros de produtividade e na composição da PEA sobre o desemprego: 1990-1999 174

Tabela 50- Efeitos totais de choques na produtividade relativa e na composição da oferta sobre as variações no desemprego por qualificação: 1990-1999 174 174

Tabela 51- Contribuições de choques relativos para o aumento do desemprego agregado e por qualificação: 1990-1999 175

$\begin{array}{ll}\text { Tabela 52- Curvas de salário por qualificação } & 175\end{array}$

Tabela 53- Efeitos de choques agregados sobre a variação do desemprego por qualificação: 1990-1999 175

Tabela 54- Variações dos parâmetros de produtividade por qualificação: 19901999

Tabela 55- Efeitos de variações nos parâmetros de produtividade e na composição da PEA sobre o desemprego: 1990-1999

176

Tabela 56- Efeitos totais de choques na produtividade relativa e na composição da oferta sobre as variações no desemprego por qualificação: 1990-1999 176

Tabela 57- Contribuições de choques relativos para o aumento do desemprego agregado e por qualificação: 1990-1999

Tabela 58- Coeficientes estimados de mudanças na demanda entre os setores 181

Tabela 59- Número de observações em cada célula coorte-período por nível de qualificação

184

Tabela 60- Resultados estimados para o desemprego relativo representando os efeitos idade, período e coorte através de polinômios 
Tabela 61- Resultados estimados para o desemprego relativo com interações 186 Tabela 62 - Resultados estimados para a probabilidade de desemprego (logit) 188

Tabela 63- Taxas de participação por idade em cada grupo de qualificação 190

Tabela 64- Taxa de participação dos homens por qualificação (\%): 1981-1999 191 Tabela 65- Taxa de participação das mulheres por qualificação (\%): 1981-1999 191

Tabela 66- Número de observações nas coortes 192

Tabela 67- Regressões logit para a probabilidade de desemprego (nãoqualificados) 193

Tabela 68- Regressões logit para a probabilidade de desemprego (semiqualificados)

194

Tabela 69 - Regressões logit para a probabilidade de desemprego (qualificados) 